\title{
Common Fixed Point Theorems of Multivalued Maps in Fuzzy Ultrametric Spaces
}

\author{
A. F. Sayed \\ Mathematics Department, Al-Lith University College, Umm Al-Qura University, P.O. Box 112, Al-Lith, \\ Makkah Al-Mukarramah 21961, Saudi Arabia \\ Correspondence should be addressed to A. F. Sayed; dr.afsayed@hotmail.com
}

Received 15 April 2013; Revised 31 August 2013; Accepted 14 September 2013

Academic Editor: Feng Feng

Copyright (C) 2013 A. F. Sayed. This is an open access article distributed under the Creative Commons Attribution License, which permits unrestricted use, distribution, and reproduction in any medium, provided the original work is properly cited.

In the setting of fuzzy ultrametric spaces, we study common fixed point theorems of multivalued maps. Our results unify, extend, and generalize some related common fixed point theorems of the literature for both ultrametric spaces (Wang and Song (2013), Gajić (2002) and (2001)) and fuzzy metric spaces (Vijayaraju and Sajath (2011)).

\section{Introduction}

In 1965, Zadeh [1] introduced the theory of fuzzy sets. Many authors introduced the notion of fuzzy metric space in different ways. George and Veeramani [2] modified the concept of fuzzy metric space introduced by Kramosil and Michalek [3] and defined Hausdorff topology in fuzzy metric space. Several authors [4-10] studied and developed the concept in different directions and proved fixed point theorems in fuzzy metric spaces. Vijayaraju and Sajath [11] extended some previous results and proved some common fixed points theorems for hybrid pair of single and multivalued maps under hybrid contractive conditions. Wang and Song [12] established some results on coincidence and common fixed point for two pairs of multivalued and single-valued maps in ultrametric spaces. In 2009, Savchenko and Zarichnyi [13] introduced the concept of fuzzy ultrametric space. Sedghi and Shobe [14] proved common fixed point theorems for self-maps satisfying contractive conditions on spherically complete fuzzy ultrametric spaces. In this paper, in the setting of fuzzy ultrametric spaces, we study common fixed point theorems of multivalued maps. Our results unify, extend, and generalize some related common fixed point theorems of the literature for both ultrametric spaces $[12,15,16]$ and fuzzy metric spaces [11].

\section{Preliminaries and Notations}

Definition 1 (see [1]). Let $X$ be any nonempty set. A fuzzy set $A$ in $X$ is a function with domain $X$ and values in $[0,1]$.
Definition 2 (see [17]). A binary operation $*:[0,1]^{2} \rightarrow$ $[0,1]$ is called a continuous triangular norm (shortly $t$-norm) if it satisfies the following conditions:

(1) * is associative and commutative,

(2) $*$ is continuous,

(3) $a * 1=a$ for all $a, b, c, d \in[0,1]$,

(4) $a * b \leq c * d$ whenever $a \leq c$ and $b \leq d$ for all $a, b, c, d \in[0,1]$.

Definition 3 (see [2]). The 3-tuple $\left(X, M,{ }^{*}\right)$ is called a fuzzy metric space if $X$ is an arbitrary (nonempty) set, $*$ is a continuous $t$-norm, and $M$ is a fuzzy set on $X^{2} \times[0, \infty)$ satisfying the following conditions, for all $x, y, z \in X$ and each $t$ and $s>0$ :

(1) $M(x, y, t)=0$,

(2) $M(x, y, t)=1$ if and only if $x=y$,

(3) $M(x, y, t)=M(y, x, t)$,

(4) $M(x, y, t) * M(y, z, t) \leq M(x, z, t+s)$,

(5) $M(x, y, \cdot):(0, \infty) \rightarrow[0,1]$ is continuous.

$M$ is called a fuzzy metric on $X$. The functions $M(x, y, t)$ denote the degree of nearness between $x$ and $y$ with respect to $t$, respectively. 
Let $\left(X, M,{ }^{*}\right)$ be a fuzzy metric space. For $t>0$, the open ball $B(x, r, t)$ with center $x \in X$ and radius $0<r<1$ is defined by

$$
B(x, r, t)=\{y \in X: M(x, y, t)>1-r\} .
$$

A subset $A \subset X$ is called open if, for each $x \in A$, there exist $t>0$ and $0<r<1$ such that $B(x, r, t) \subset A$. Let $\tau$ denote the family of all open subsets of $X$. Then $\tau$ is a topology on $X$ induced by the fuzzy metric $M$. This topology is Hausdorff and first countable.

Definition 4 (see [18]). Let $(X, d)$ be a metric space. If the metric $d$ satisfies strong triangle inequality

$$
d(x, y) \leq \max \{d(x, z), d(z, y)\}, \quad \forall x, y, z \in X,
$$

then $d$ is called an ultrametric on $X$ and the pair $(X, d)$ is called an ultrametric space.

Definition 5 (see [18]). An ultrametric space $(X, d)$ is said to be spherically complete if every shrinking collection of balls in $X$ has a nonempty intersection.

Definition 6 (see $[14])$. Let $\left(X, M,{ }^{*}\right)$ be a fuzzy metric space. If the fuzzy metric $M$ satisfies strong triangle inequality

$$
\begin{array}{r}
M(x, y, t) \geq \min \{M(x, z, t), M(z, y, t)\}, \\
\forall x, y, z \in X, t>0,
\end{array}
$$

then $M$ is called a fuzzy ultrametric on $X$ and the 3-tuple $\left(X, M,{ }^{*}\right)$ is called a fuzzy ultrametric space.

Definition 7 (see [14]). A fuzzy ultrametric space $\left(X, M,{ }^{*}\right)$ is said to be spherically complete if every shrinking collection of balls in $X$ has a nonempty intersection.

Remark 8 (see [14]). (i) Let $d$ be an ultrametric on $X$ and $a *$ $b=a \cdot b$ for all $a, b \in[0,1]$. For each $t \in(0, \infty)$, define

$$
M(x, y, t)=\left(\frac{1}{1+d(x, y)}\right)^{\psi(t)},
$$

for all $x, y, z \in X, t>0$, and $\psi \in \Psi$. Then fuzzy metric $M$ is also a fuzzy ultrametric.

(ii) Let an ultrametric space $(X, d)$ be spherically complete and $a * b=a \cdot b$ for all $a, b \in[0,1]$. For each $t \in(0, \infty)$, define

$$
M(x, y, t)=\left(\frac{1}{1+d(x, y)}\right)^{\psi(t)},
$$

for all $x, y, z \in X, t>0$, and $\psi \in \Psi$. Then fuzzy metric space $\left(X, M,{ }^{*}\right)$ is also spherically complete.

\section{Main Results}

Let $\left(X, M,{ }^{*}\right)$ be a fuzzy ultrametric space. Let $\mathrm{CB}(X)$ denote the set of all nonempty closed bounded subsets of $X$. For every $A, B, C \in \mathrm{CB}(X)$ and $t>0$, we define

$$
M_{\nabla}(A, B, t)=\min \left\{\bigwedge_{a \in A} M^{\nabla}(a, B, t), \bigwedge_{b \in B} M^{\nabla}(b, A, t)\right\},
$$

where $M^{\nabla}(x, C, t)=\bigvee_{c \in C} M(x, c, t)$.
Remark 9 (see [8]). It is obvious that, for all $A, B \in \mathrm{CB}(X)$ and $t>0$, we have

(i) $M_{\nabla}(A, B, t) \leq M^{\nabla}(a, B, t)$, whenever $a \in A$,

(ii) $M_{\nabla}(A, B, t)=1$ if and only if $A=B$.

It is easy to prove that $\left(\mathrm{CB}(X), M_{\nabla},{ }^{*}\right)$ is a Hausdorff ultrametric space.

Definition 10. Let $\left(X, M,{ }^{*}\right)$ be a fuzzy ultrametric space. An element $x \in X$ is said to be a coincidence point of a multivalued map $T: X \rightarrow \mathrm{CB}(X)$ and a single-valued map $f: X \rightarrow X$ if $f x \in T X$. We denote

$$
C(f, T)=\{x \in X \mid f x \in T x\}
$$

the set of coincidence points of a multivalued map $T$ and single-valued map $f$.

Definition 11. Let $\left(X, M,{ }^{*}\right)$ be a fuzzy ultrametric space, $T$ : $X \rightarrow \mathrm{CB}(X)$ a multivalued map, and $f: X \rightarrow X$ if $f x \in T X$ is a single-valued map. $T$ and $f$ are said to be coincidentally computing at $z \in X$ if $f z \in T z \Rightarrow f T z \subseteq T f z$.

Definition 12. Let $\left(X, M,{ }^{*}\right)$ be a fuzzy ultrametric space. An element $x \in X$ is said to be a common fixed point of two multivalued maps $S, T: X \rightarrow \mathrm{CB}(X)$ and a single-valued map $f: X \rightarrow X$ if

$$
x=f x \in S x \cap T x
$$

Theorem 13. Let $\left(X, M,{ }^{*}\right)$ be a fuzzy ultrametric space. Let $S, T: X \rightarrow C B(X)$ be a pair of multivalued maps and $f, g:$ $X \rightarrow X$ a pair of single-valued maps satisfying the following conditions:

(a) $f g(x)$ is spherically complete;

(b) $M_{\nabla}(S x, T y, t)>\min \left\{M(f x, g y, t), M^{\nabla}(f x, S x, t)\right.$, $\left.M^{\nabla}(g y, T y, t)\right\}$, for all $x, y \in X$, with $f x \neq g y$, and for $t>0$;

(c) $f S=S g, f g=g f, f T=T f, g S=S g, g T=T g$, $S T=T S$;

(d) If $S(X) \subseteq f(X), T(X) \subseteq g(X)$, then there exist points $u$ and $v$ in $X$, such that

$$
f u \in S u, \quad g v \in T v, \quad f u=g v, \quad S u=T v .
$$

Proof. Let $B_{a}=\left(f g a ; \min \left\{M^{\nabla}(f g a, S g a, t), M^{\nabla}(f g a, T f a, t)\right\}\right)$ denote the closed ball with centered $f g a$ and radius

$$
\min \left\{M^{\nabla}(f g a, S g a, t), M^{\nabla}(f g a, T f a, t)\right\} .
$$

Let $\mathbf{A}$ be the collection of all the spheres for all $a \in f g(X)$. Then the relation

$$
B_{a} \leq B_{b} \text { if } B_{b} \subseteq B_{a}
$$

is a partial order on $\mathbf{A}$. 
Consider a totally ordered subfamily $\mathbf{A}_{1}$ of A. Since $f g(X)$ is spherically complete, we have

$$
\bigcap_{B_{a} \in \mathbf{A}_{1}} B_{a}=B \neq \phi .
$$

Let $f g b \in B$, where $b \in f g(X)$ and $B_{a} \in \mathbf{A}_{1}$. Then $f g b \in$ $B_{a}$. Hence

\section{$M(f g b, f g a, t)$}

$$
\geq \min \left\{M^{\nabla}(f g a, S g a, t), M^{\nabla}(f g a, T f a, t)\right\} .
$$

If $a=b$, then $B_{a}=B_{b}$. Assume that $a \neq b$. Let, for all $x \in B_{b}$,

$$
\begin{aligned}
& M(x, f g b, t) \\
& \quad \geq \min \left\{M^{\nabla}(f g b, S g b, t), M^{\nabla}(f g b, T f b, t)\right\} .
\end{aligned}
$$

Since Sga is a nonempty closed bounded set, then there exists an element $p \in S g a$, such that

$$
M^{\nabla}(f g a, S g a, t)=M(f g a, p, t) .
$$

$T f a$ is a nonempty closed bounded set; then there exists an element $q \in T f a$ such that

$$
\begin{gathered}
M^{\nabla}(f g a, T f a, t)=M(f g a, q, t), \\
\min \left\{M^{\nabla}(f g b, S g b, t), M^{\nabla}(f g b, T f b, t)\right\} \\
=\min \left\{\bigvee_{c \in S g b} M(f g b, c, t), \bigvee_{d \in T g b} M(f g b, d, t)\right\} \\
\geq \min \{M(f g b, f g a, t), M(f g a, q, t), \\
\bigvee_{c \in S g b} M(q, c, t), M(f g b, f g a, t), \\
\left.M(f g a, p, t), \bigvee_{d \in T f b} M(p, d, t)\right\} \\
\geq \min \left\{M(f g b, f g a, t), M^{\nabla}(f g a, T f a, t),\right. \\
M_{\nabla}(T f a, S g b, t), M(f g b, f g a, t), \\
\left.M^{\nabla}(f g a, S g a, t), M_{\nabla}(S g a, T f b, t)\right\}
\end{gathered}
$$

$$
\begin{gathered}
>\min \left\{M(f g b, f g a, t), M^{\nabla}(f g a, T f a, t),\right. \\
M^{\nabla}(f g a, S g a, t), \\
\min \left\{M(f g b, f g a, t), M^{\nabla}(f g a, T f a, t),\right. \\
\left.\left.M^{\nabla}(f g b, S g b, t)\right\}\right\}, \\
\min \left\{M(f g a, f g b, t), M^{\nabla}(f g a, S g a, t),\right. \\
\left.M^{\nabla}(g f b, T f b, t)\right\} \\
\geq \min \left\{M^{\nabla}(f g a, T f a, t), M^{\nabla}(f g a, S g a, t)\right\} .
\end{gathered}
$$

From the two conditions (a), (b) and (13), we now have $M(x, f g a, t)$

$$
\begin{aligned}
& \geq \min \{M(x, f g b, t), M(f g b, f g a, t)\} \\
& \geq \min \left\{M^{\nabla}(f g a, S g a, t), M^{\nabla}(f g a, T f a, t)\right\} .
\end{aligned}
$$

So $x \in B_{a}$; we have just proved that $B_{b} \subseteq B_{a}$, for every $B_{a} \in \mathbf{A}_{1}$. Thus $B_{b}$ is an upper bound in $\mathbf{A}$ for the family $\mathbf{A}_{1}$, and hence by Zorn's lemma, there is a maximal element in $\mathbf{A}$, say $B_{Z}, z \in f g(X)$. There exists an element $w \in X$ such that $z=f g w$.

Suppose

$$
\begin{aligned}
& f(g f g w) \notin S(g f g w), \\
& g(f f g w) \notin T(f f g w) .
\end{aligned}
$$

Since Sgfgw and Tffgw are nonempty closed bounded sets, then there exist $k \in S g f g w$ and $l \in T f f g w$ such that

$$
\begin{aligned}
& M^{\nabla}(f g f g w, S g f g w, t)=M(f g f g w, k, t), \\
& M^{\nabla}(f g f g w, T f f g w, t)=M(f g f g w, l, t) .
\end{aligned}
$$

From the two conditions (b), (c) and (19), we have

$M^{\nabla}(\operatorname{Sgfgw}, T S f g w, t)$

$$
\begin{aligned}
& =\bigvee_{e \in T S f g w} M(g S f g w, e, t) \\
& \geq \min \left\{M^{\nabla}(S g f g w, f g f g w, t),\right.
\end{aligned}
$$

$$
\left.M(f g f g w, k, t), \bigvee_{e \in T S f g w} M(k, e, t)\right\}
$$

$\geq \min \left\{M^{\nabla}(S g f g w, f g f g w, t)\right.$,

$\left.M_{\nabla}(S g f g w, T S f g w, t)\right\}$

$>\min \left\{M^{\nabla}(S g f g w, f g f g w, t)\right.$,

$\min \{M(f g f g w, f S f g w, t)$, 


$$
\begin{gathered}
M^{\nabla}(f g f g w, S g f g w, t), \\
\left.\left.M^{\nabla}(g S f g w, T S f g w, t)\right\}\right\} \\
=M^{\nabla}(f g f g w, S g f g w, t), \\
M^{\nabla}(T f f g w, S T f g w, t) \\
=\bigvee_{h \in S T f g w} M(f T f g w, h, t) \\
\geq \min \left\{M^{\nabla}(T f f g w, f g f g w, t),\right. \\
\left.M_{\nabla}(T f f g w, S T f g w, t)\right\} \\
>\min \left\{M^{\nabla}(T f f g w, g f f g w, t),\right. \\
\min \{(f T f g w, g f f g w, t), \\
M^{\nabla}(f T f g w, S T f g w, t), \\
\left.\left.M^{\nabla}(g f f g w, T f f g w, t)\right\}\right\} \\
=M^{\nabla}(T f f g w, f g f g w, t) .
\end{gathered}
$$

From the two conditions (b), (c) and (19)-(22), we have

$$
\begin{aligned}
& M^{\nabla}(f g g S w, S g g S w, t) \\
& =\bigvee_{m \in S g g S w} M(f g g S w, m, t) \\
& \geq \min \left\{M^{\nabla}(f g g S w, T S f g w, t),\right. \\
& M(T S f g w, T f f g w, t), \\
& M^{\nabla}(T f f g w, f g f g w, t), \\
& \left.M(g f f g w, l, t), \bigvee_{m \in S g g S w} M(l, m, t)\right\} \\
& \geq \min \left\{M^{\nabla}(f g f g w, S g f g w, t),\right. \\
& M^{\nabla}(f g f g w, T f f g w, t), \\
& \min \{M(f g g s w, g f f g w, t), \\
& M^{\nabla}(f g g S w, S g g S w, t), \\
& \left.\left.M^{\nabla}(g f f g S w, T f f g w, t)\right\}\right\} \\
& \geq \min \left\{M^{\nabla}(f g f g w, S g f g w, t),\right. \\
& \left.M^{\nabla}(f g f g w, T f f g w, t)\right\},
\end{aligned}
$$

$M^{\nabla}(f g f T w, T f f T w, t)$

$$
=\bigvee_{n \in T f f T w} M(f g f T w, n, t)
$$$$
\geq \min \left\{M^{\nabla}(f g f T w, S T f g w, t),\right.
$$$$
M(S T f g w, S g f g w, t),
$$$$
M^{\nabla}(S g f g w, f g f g w, t),
$$$$
\left.M(f g f g w, k, t), \bigvee_{n \in T f f T w} M(k, n, t)\right\}
$$$$
\geq \min \left\{M^{\nabla}(f g f g w, T f f g w, t),\right.
$$$$
M^{\nabla}(f g f g w, S g f g w, t),
$$$$
\left.M_{\nabla}(S g f g w, T f f T w, t)\right\}
$$$$
\geq \min \left\{M^{\nabla}(f g f g w, T f f g w, t),\right.
$$$$
M^{\nabla}(f g f g w, S g f g w, t),
$$$$
\min \{M(f g f g w, g f f T w, t),
$$$$
M^{\nabla}(f g f g w, S g f g w, t),
$$$$
\left.\left.M^{\nabla}(g f f T w, T f f T w, t)\right\}\right\}
$$

$\geq \min \left\{M^{\nabla}(f g f g w, T f f g w, t)\right.$,

$$
\left.M^{\nabla}(f g f g w, S g f g w, t)\right\} .
$$

From (21) and (23), we have

$$
\begin{gathered}
\min \left\{M(S g f g w, T S f g w, t), M^{\nabla}(f g g S w, S g g S w, t)\right\} \\
>\min \left\{M^{\nabla}(f g f g w, S g f g w, t),\right. \\
\left.M^{\nabla}(f g f g w, T f f g w, t)\right\} .
\end{gathered}
$$

From (22) and (3.7), we have

$$
\begin{aligned}
& \min \left\{M(S T f g w, T f f g w, t), M^{\nabla}(f g f T w, T f f T w, t)\right\} \\
& >\min \left\{M^{\nabla}(f g f g w, T f f g w, t),\right. \\
& \left.\qquad M^{\nabla}(f g f g w, S g f g w, t)\right\} . \\
& \text { If } \min \left\{M^{\nabla}(f g f g w, S g f g w, t), M^{\nabla}(f g f g w, T f f g w, t)\right\} \\
& =M^{\nabla}(f g f g w, S g f g w, t),
\end{aligned}
$$

then from (24), we have $f g f g w \notin B_{g S w} \Rightarrow f g z \notin B_{g S w}$. 
Hence $B_{z} \not \subset B_{g S w}$. It is a contradiction to the maximality of $B_{z}$ in $\mathbf{A}$, since $g S w \subseteq g f(X)=f g(X)$.

$$
\text { If }
$$

$$
\begin{aligned}
\min & \left\{M^{\nabla}(f g f g w, S g f g w, t), M^{\nabla}(f g f g w, T f f g w, t)\right\} \\
& =M^{\nabla}(f g f g w, T f f g w, t),
\end{aligned}
$$

then from (25), we have $f g f g w \notin B_{f T w} \Rightarrow f g z \notin B_{f T w}$.

Hence $B_{z} \not \subset B_{f T w}$. It is a contradiction to the maximality of $B_{z}$ in $\mathbf{A}$, since $f T w \subseteq f g(X)$,

$$
f(g f g w) \in S(f f g w), \quad g(g f g w) \in T(f f g w) .
$$

In addition, $f(g f g w)=g(f f g w)$.

Using the two conditions (b), (c) and (28), we obtain

$$
M_{\nabla}(S g f g w, T f f g w, t)
$$

$$
\begin{gathered}
>\min \left\{M(f g f g w, g f f g w, t), M^{\nabla}(f g f g w, S g f g w, t),\right. \\
\left.M^{\nabla}(g f f g w, T f f g w, t)\right\}=1 .
\end{gathered}
$$

Hence $S(g f g w)=T(f f g w)$. Then the proof is completed.

Theorem 14. Let $\left(X, M,{ }^{*}\right)$ be a fuzzy ultrametric space. Let $S, T: X \rightarrow C B(X)$ be a pair of multivalued maps and let $f: X \rightarrow X$ be a single-valued map satisfying the following conditions:

(a) $f(x)$ is spherically complete;

(b) $M_{\nabla}(S x, T y, t)>\min \left\{M(f x, f y, t), M^{\nabla}(f x, S x, t)\right.$, $\left.M^{\nabla}(f y, T y, t)\right\}$, for all $x, y \in X$, with $x \neq y$, and for $t>0$

(c) $f S=S f, f T=T f, S T=T S$;

(d) If $S(X) \subseteq f(X), T(X) \subseteq f(X)$.

Then $f, S$, and T have a coincidence point in X. Moreover, if $f$ and $S, f$ and $T$ are coincidentally commuting at $z \in$ $C(f, T)$ and $f f z=f z$, then $f, S$, and $T$ have a common fixed point in $X$.

Proof. If $f=g$ in Theorem 13, we obtain that there exist points $u$ and $v$ in $X$ such that

$$
f u \in S u, \quad f v \in T v, \quad f u=f v, \quad S u=T v .
$$

As $u \in C(f, S), f$ and $S$ are coincidentally commuting at $u$ and $f f u=f u$.

Writing $w=f u$, then $w \in S u, w \in T v$. Then we have

$$
\begin{gathered}
f w=w, \\
w=f w \in f(S u) \subseteq S(f u)=S w .
\end{gathered}
$$

Now, since also $u \in C(f, T), f$ and $T$ are coincidentally commuting at $u$ and $f f u=f u$, so we obtain

$$
w=f w \in f(T v) \subseteq T(f v)=T w .
$$

Thus, we have proved that $w=f w \in S w \cap T w$; that is, $w$ is a common fixed point of $f, S$, and $T$.
The proof of the following corollary is easy, so it is omitted.

Corollary 15. Let $\left(X, M,{ }^{*}\right)$ be a spherically complete fuzzy ultrametric space. Let $S, T: X \rightarrow C B(X)$ be a pair of multivalued maps satisfying the following conditions:

(a) $M_{\nabla}(S x, T y, t)>\min \left\{M(x, y, t), M^{\nabla}(x, S x, t), M^{\nabla}(y\right.$, $T y, t)\}$, for all $x, y \in X$, with $x \neq y$, and for $t>0$;

(b) $S T=T S$.

Then, there exists a point $z$ in $X$ such that $z \in S z \cap T z$ and $S z=T z$.

Now we give an example to illustrate Theorem 13.

Example 16. Let $\left(X, M,{ }^{*}\right)$ be a fuzzy ultrametric space in which $X=[0,1], a * b=\min \{a, b\}$ for all $a, b \in[0,1]$, and

$$
M(x, y, t)=\frac{t}{t+|x-y|}
$$

for all $t>0$.

Define the maps $S, T, f, g$ on $X$ as follows:

$$
\begin{array}{r}
S x=T x=1, \quad f x=\frac{x+1}{2}, \quad g x=\frac{2 x+1}{3}, \\
\forall x \in X .
\end{array}
$$

Define

$$
W\left(t_{1}, t_{2}, t_{3}, t_{4}, t_{5}\right)=t_{1}-\left(\min \left\{t_{2}, t_{3}, t_{4}, t_{5}\right\}\right)^{h},
$$

where $W$ is a function from $[0,1]^{5} \rightarrow[-1,1]$ satisfying the following conditions:

$\left(W_{1}\right) W\left(t_{1}, \ldots, t_{5}\right)$ is increasing in $t_{1}$ and decreasing in $t_{2}, \ldots, t_{5}$

$\left(W_{2}\right) W(u, v, v, v, v) \geq$,0 implies that $u>v$, for all $u \in$ $[0,1]$ and $v \in[0,1)$.

Then for any $h \in(0,1)$, the following inequality

$$
\begin{gathered}
M_{\nabla}(S x, T y, t) \\
>\left(\operatorname { m i n } \left\{M(f x, g y, t), M^{\nabla}(f x, S x, t),\right.\right. \\
\left.\left.M^{\nabla}(g y, T y, t)\right\}\right)^{h}
\end{gathered}
$$

is satisfied for all $x, y \in X$, with $x \neq y$, and for all $t>0$ since the LHS of the inequality is 1 .

Clearly all conditions in Theorem 13 are satisfied, so 1 is the unique common fixed point of $S, T, f$, and $g$.

\section{Conclusion}

In this paper, we get coincidence point theorems and common fixed point theorems for two pairs of multivalued and single-valued maps satisfying different contractive conditions on spherically complete fuzzy ultrametric space, which are generalized results for both ultrametric spaces $[12,15,16]$ and fuzzy metric spaces [11]. 


\section{Acknowledgment}

The author is thankful to the referees for their valuable suggestions in preparing this paper.

\section{References}

[1] L. A. Zadeh, "Fuzzy sets," Information and Control, vol. 8, no. 3, pp. 338-353, 1965.

[2] A. George and P. Veeramani, "On some results in fuzzy metric spaces,” Fuzzy Sets and Systems, vol. 64, no. 3, pp. 395-399, 1994.

[3] I. Kramosil and J. Michalek, "Fuzzy metric and statistical metric spaces," Kybernetika, vol. 11, no. 5, pp. 336-344, 1975.

[4] M. Abbas, I. Altun, and D. Gopal, "Comon fixed points theorems for non compatible mappings spaces in fuzzy metric spaces," Bulletin of Mathematical Analysis and Applications, vol. 1, no. 2, pp. 47-56, 2009.

[5] Z. K. Deng, "Fuzzy pseudo-metric spaces," Journal of Mathematical Analysis and Applications, vol. 86, no. 1, pp. 74-95, 1982.

[6] M. Grabiec, "Fixed points in fuzzy metric spaces," Fuzzy Sets and Systems, vol. 27, no. 3, pp. 385-389, 1988.

[7] O. Kaleva and S. Seikkala, "On fuzzy metric spaces," Fuzzy Sets and Systems, vol. 12, no. 3, pp. 215-229, 1984.

[8] I. Kubiaczyk and S. Sharma, "Common coincidence point in fuzzy metric spaces," Journal of Fuzzy Mathematics, vol. 11, no. 1, pp. 1-5, 2003.

[9] S. Sharma, "Common fixed point theorems in fuzzy metric spaces," Fuzzy Sets and Systems, vol. 127, no. 3, pp. 345-352, 2002.

[10] B. Singh and M. S. Chauhan, "Common fixed points of compatible maps in fuzzy metric spaces," Fuzzy Sets and Systems, vol. 115, no. 3, pp. 471-475, 2000.

[11] P. Vijayaraju and Z. M. I. Sajath, "Common fixed points of single and multivalued maps in fuzzy metric spaces," Applied Mathematics, vol. 2, pp. 595-599, 2011.

[12] Q. Wang and M. Song, "Common fixed point theorems of multivalued maps in ultra metric space," Applied Mathematics, vol. 4, pp. 417-420, 2013.

[13] A. Savchenko and M. Zarichnyi, "Fuzzy ultrametrics on the set of probability measures," Topology, vol. 48, no. 2-4, pp. 130-136, 2009.

[14] S. Sedghi and N. Shobe, "Coincidence and fixed point theorems in fuzzy ultrametric spaces," Azerbaijan Journal of Mathematics, vol. 3, no. 1, pp. 2218-6816, 2013.

[15] L. Gajić, "A multivalued fixed point theorem in ultrametric spaces," Matematicki Vesnik, vol. 54, no. 3-4, pp. 89-91, 2002.

[16] L. Gajić, "On ultrametric space," Novi Sad Journal of Mathematics, vol. 31, no. 2, pp. 69-71, 2001.

[17] B. Schweizer and A. Skala, "Statistical metric spaces," Pacific Journal of Mathematics, vol. 10, pp. 314-334, 1960.

[18] A. C. M. Van Rooij, Non-Archimedean Functional Analysis, Marcel Dekker, New York, NY, USA, 1973. 


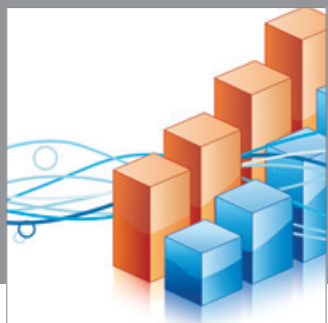

Advances in

Operations Research

mansans

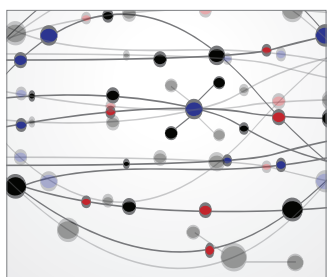

The Scientific World Journal
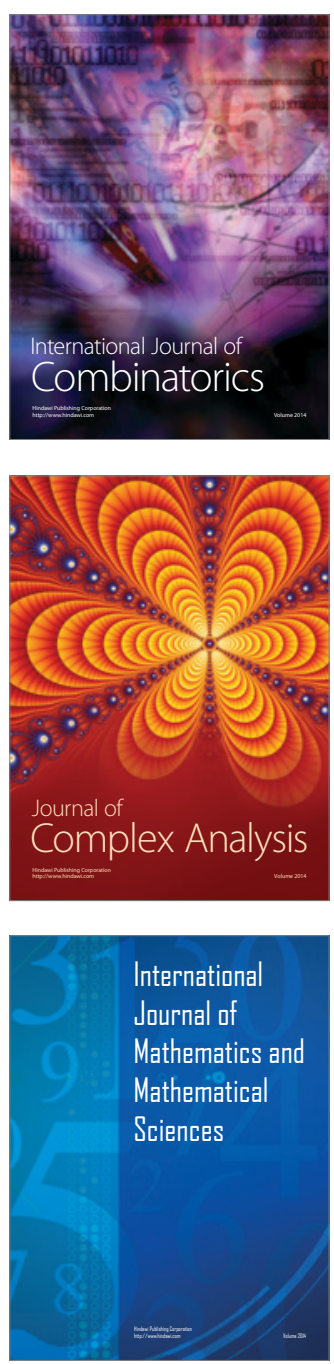
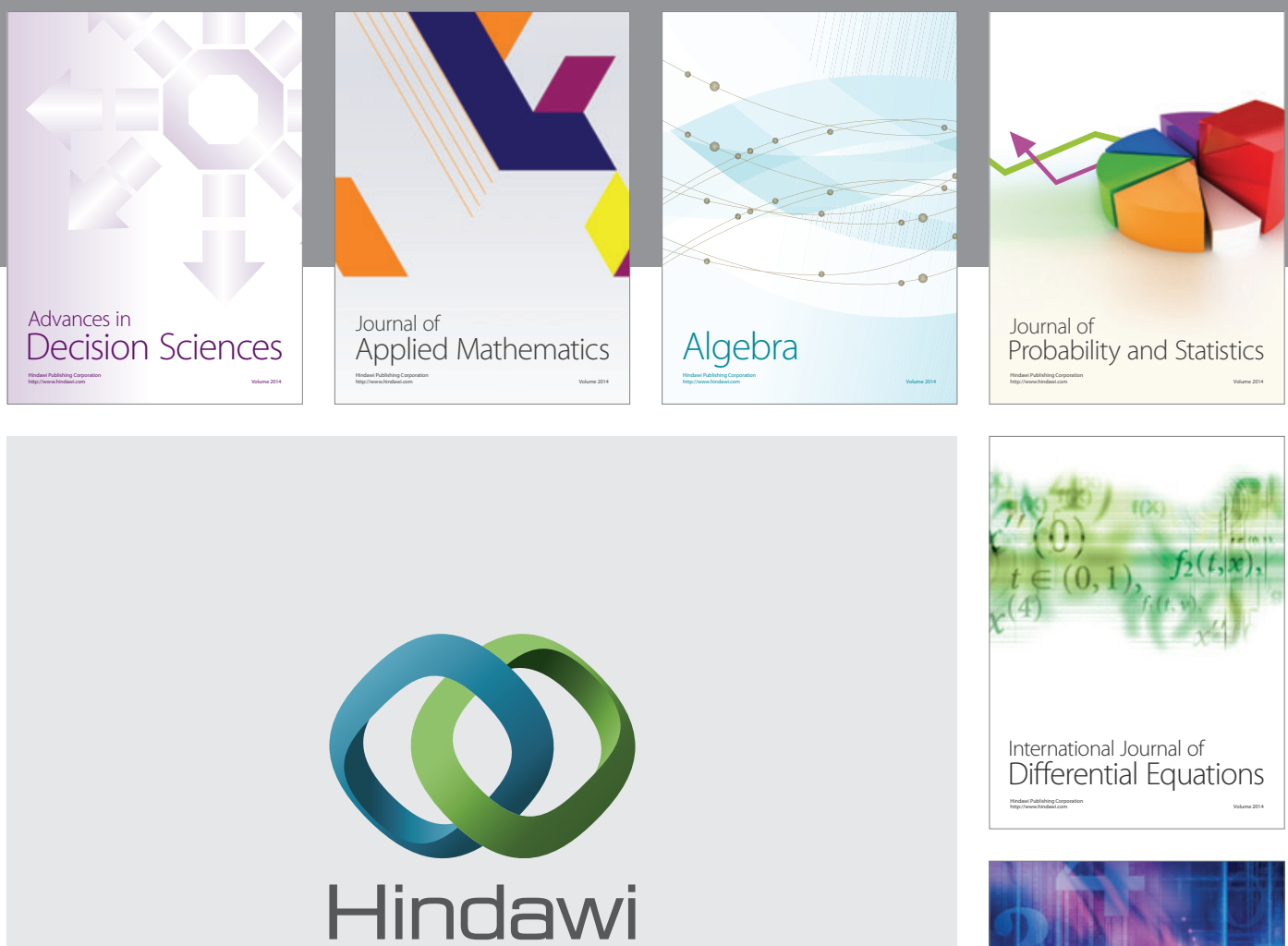

Submit your manuscripts at http://www.hindawi.com
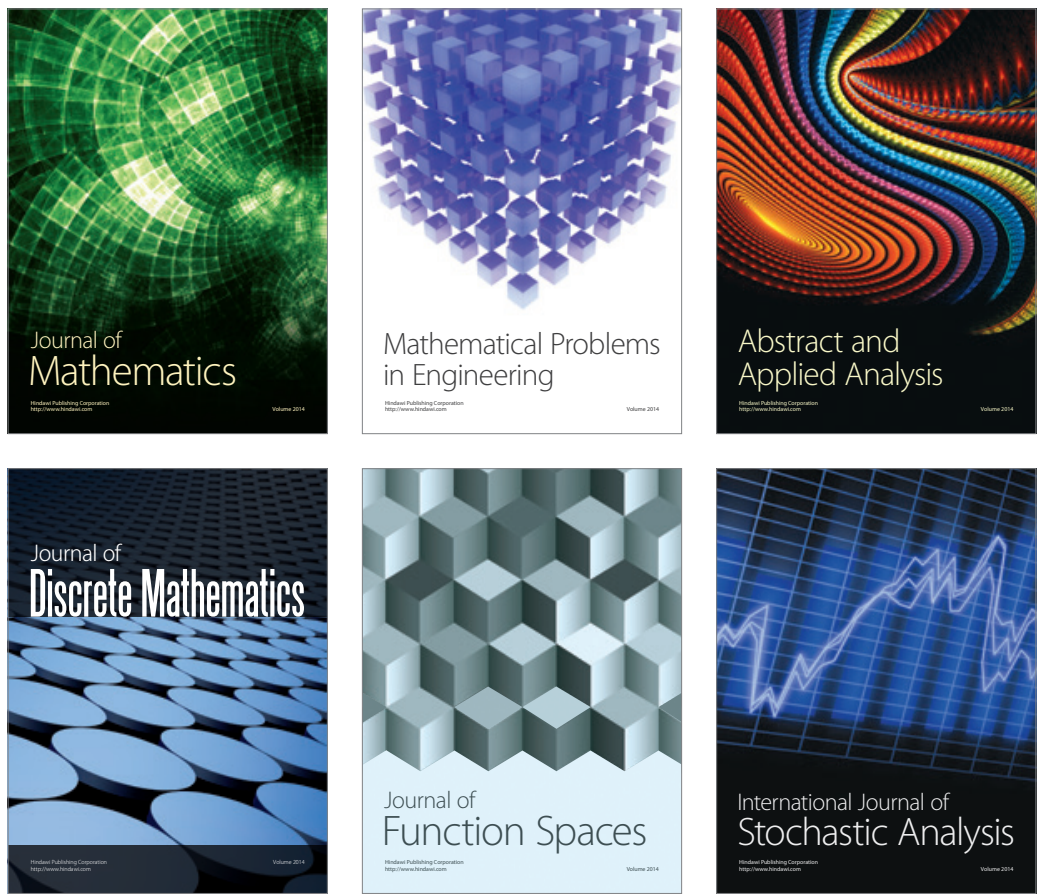

Journal of

Function Spaces

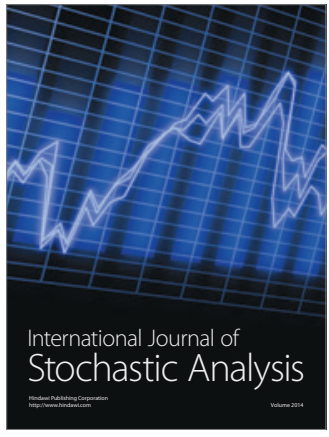

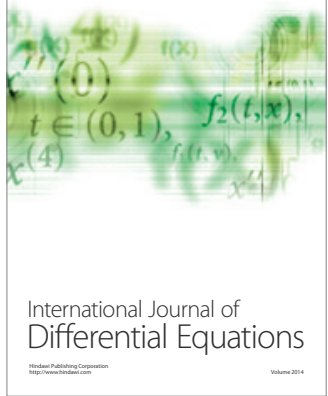
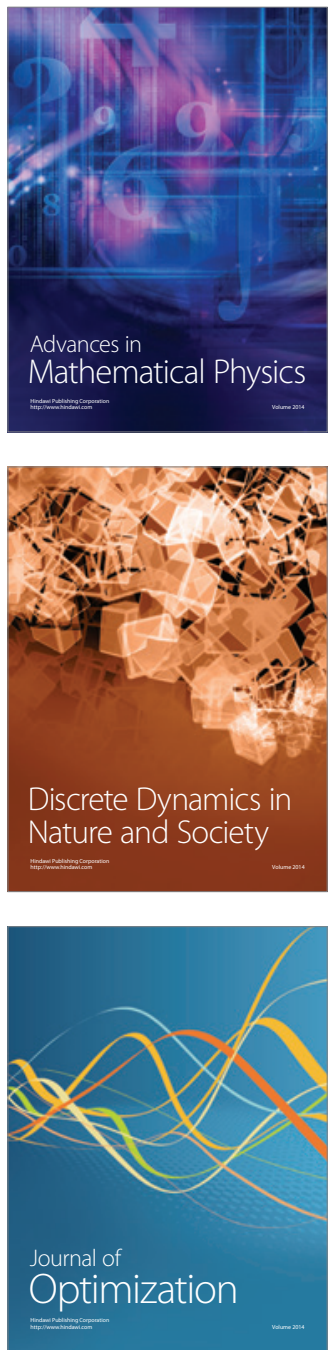